\title{
Characterization of the Indoor-to-Outdoor Wireless Channel in Air-to-Ground Communication Systems
}

\author{
Lorenzo Norberti \\ DEIB, Politecnico di Milano \\ Milan, Italy \\ lorenzo.norberti@mail.polimi.it
}

\author{
Roberto Nebuloni \\ IEIIT, Consiglio Nazionale delle Ricerche \\ Milan, Italy \\ roberto.nebuloni@ieiit.cnr.it
}

\author{
Maurizio Magarini \\ DEIB, Politecnico di Milano \\ Milan, Italy \\ maurizio.magarini@polimi.it
}

\begin{abstract}
Wireless communication between User Terminals (UTs) inside a building and an outdoor base station mounted onboard an unmanned aerial vehicle (UAV) is receiving a higher interest in emergency management scenarios and where users require on demand high throughput services. In such applications, a fundamental aspect is the thorough characterization of the propagation environment through parameters such as the UTUAV distance and the number of walls and floors crossed. In this paper, we characterize the indoor-to-outdoor wireless channel by using a commercial ray-tracing software. The reference scenario is a four-floor building. The UTs are uniformly distributed within each floor and two UAV positions are considered nearby the building. As a main contribution, we present numerical results in terms of path loss against the UT-UAV distance. The dependence of the path loss on the number of floors between the UT and the UAV is highlighted as well. Finally, the ray-tracing results are compared with those predicted by a few available propagation models.
\end{abstract}

Index Terms-Ray-tracing, indoor-to-outdoor wireless channel, air-to-ground communication, UAV, path loss, propagation.

\section{INTRODUCTION}

In the context of wireless communications for emergency services, there is an increasing interest in the development of solutions that consider the use of unmanned aerial vehicles (UAVs) to support the connectivity in the case where the traditional network infrastructure is not available [1]. Typical cases are those where a mini base-station (BS) is mounted on-board the UAV [2] or where the UAV acts as a relay [3].

In emergency scenarios the users can be located both in outdoor or indoor environments. In particular, for users trapped inside buildings there is the need to establish a stable indoor-to-outdoor communication link that can guarantee a timely and reliable transmission with the emergency command center [4]. Therefore, a viable solution for next generation emergency wireless networks [4] could be the usage of a UAV flying nearby the external walls of a building. The obvious advantage of a UAV-based link over a terrestrial link is that the BS position can be optimized according to service-specific requirements. On the other side, UAVs will play an important role in fifth generation (5G) networks compared to fixed infrastructure communications due to their prominent attributes, such as flexible deployment and additional characteristic of controlled mobility [5].

In this paper, we study the propagation channel of an airto-ground (A2G) communication link between a user terminal
(UT) inside a building and an outdoor UAV hovering close to the building. The main motivation for our study is the scarcity of channel measurements and models for this specific environment [6]. A major reason for the lack of modelling is that different wireless technologies are used in indoor and outdoor environments, i.e., WiFi for indoor coverage and wireless cellular for outdoor, respectively. A previous study about the characterization of the indoor-to-outdoor propagation can be found in [7], which focuses on terrestrial networks. The approach is based on ray-tracing and exhaustive measurements campaign and was conducted to develop a unified model for the interference in $5 \mathrm{G}$ networks where femtocells underlay macrocells. However, the paradigm is changing fast due to the new services offered by $5 \mathrm{G}$ cellular technology, which includes the development of vertical aerial networks [5].

In this work, we analyze the indoor-to-outdoor propagation channel through ray tracing simulation [8]. As is well known, ray-tracing methods are used to obtain high accuracy in indoor [9], or outdoor-only environments [10], [11], [12]. In such scenarios, the penetration of the signal through the external walls of the buildings is usually neglected for simplicity. Simulations have been carried out by the commercial ray-tracing software Wireless Insite [13], which is briefly described in Sec. II. The reference scenario is a four-floor building with users uniformly distributed within each floor (Sec. III). In Sec. IV, we present the results of ray-tracing simulations in terms of path-loss as a function of the distance between the UT and the UAV and of the corresponding difference in height. Moreover, we compare our numerical results with pathloss values predicted by a few models available in the open literature. Finally, conclusions are drawn in Sec. V.

\section{RAY-TRACING SIMULATION}

The indoor-to-outdoor wireless communication channel represents a complex propagation environment, which prompts for the development of suitable channel models. To this aim, empirical models based on measurements may be too simple not taking into account all the relevant parameters or may be too specific depending on the underlying measurement dataset. A good way to proceed is to resort to ray-tracing approaches. In general, the advantage of simulations is that they permit to study the dependence of channel features on general link parameters (i.e., transmitter-receiver distance and frequency), 


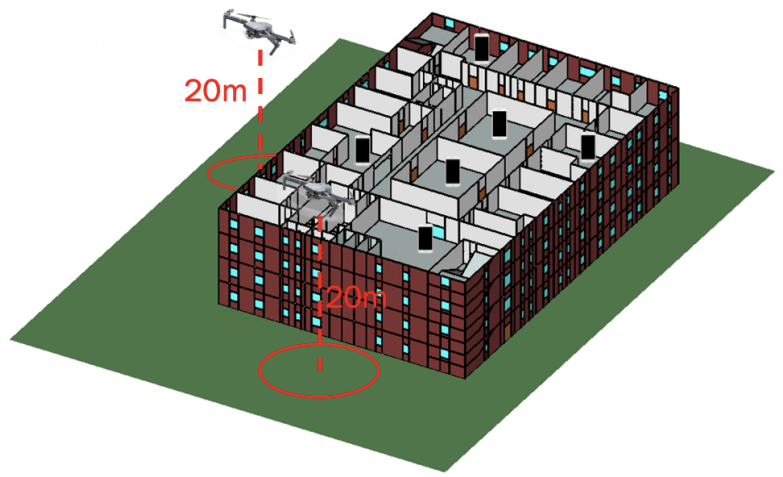

Fig. 1. Simulated scenario. The transmitters are randomly displaced inside a four-floor office building. The two receivers are on-board as many UAVs.

TABLE I

CHARACTERISTICS OF USED MATERIALS AND OTHER SIMULATION SETTINGS.

\begin{tabular}{|l||l|l|l|}
\hline Material & Permittivity & Conductivity & Thickness \\
\hline Brick & 4.44 & $0.001 \mathrm{~S} / \mathrm{m}$ & $0.25 \mathrm{~m}$ \\
\hline Concrete & 15 & $0.015 \mathrm{~S} / \mathrm{m}$ & $0.3 \mathrm{~m}$ \\
\hline Wood & 5 & 0 & $0.03 \mathrm{~m}$ \\
\hline Glass & 2.4 & 0 & $0.003 \mathrm{~m}$ \\
\hline Layered Drywall & 2.8 & $0.001 \mathrm{~S} / \mathrm{m}$ & $0.125 \mathrm{~m}$ \\
\hline \hline Waveform & Type & Frequency & \\
\hline & Sinusoidal & $1.8 \mathrm{GHz}$ & \\
\hline \hline Antenna & Type & Polarization & \\
\hline & Isotropic & Horizontal & \\
\hline
\end{tabular}

but also to evaluate the effect of scenario-specific elements (e.g., building materials, floors and walls).

Ray-tracing products available on the market include advanced options to generate complex environments and allow an accurate reconstruction of the electromagnetic field by implementation of full 3D methods. Ray-tracing is especially suitable to model wireless communication links including indoor environments, where the signal propagates through multiple paths due to reflection and transmission through floors, walls, ceilings, windows, etc. As a result, a number of contributions can reach the receiver, each one with its own peculiarities [14].

The commercial 3D ray-tracing simulation software Wireless InSite was used here to calculate the electromagnetic field transmitted by UTs located inside a building that is received by two UAVs hovering close to its external walls. We used the Wireless InSite X3D model, which implements an Exact Path Calculator (EPC) algorithm and supports multicore processing [13, p. 233]. The EPC reduces errors by adapting the interaction points (i.e., reflections, diffractions and transmissions) and checking that the diffraction and reflection angles are valid. The output data are stored into text files enumerating up to 25 rays received by each UAV from every mobile terminal, their characteristics (received power, angle of arrival and distance travelled), as well as the history of each ray (i.e., the sequence of reflections, transmissions and diffractions). The above data were subsequently processed by

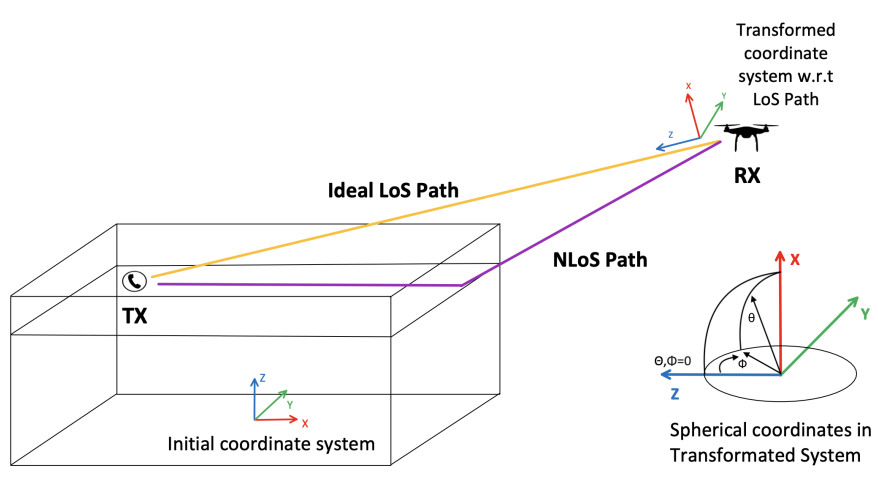

Fig. 2. Coordinate system.

Matlab to extract the received power and the path loss across every possible link.

\section{Single Building Scenario}

A four-floor office building scenario was generated by the Floor Plan Editor tool available in Wireless InSite, as shown in Fig. 1. The building has been generated by replicating four times a one-floor rectangular-shaped $60 \times 40 \mathrm{~m}$ structure with height of $4 \mathrm{~m}$ to achieve a total height of $16 \mathrm{~m}$.

The thickness and the electromagnetic properties of internal and external walls, ceilings, windows and doors, as provided by the Wireless InSite data base, are listed in Table I. Internal walls are made of layered drywall, whereas the external walls are bricks and concrete is assumed for ceilings. The thickness is an important parameter as it affects the transmission coefficient through an obstacle [15].

A population of 100 users was uniformly distributed in each of the four floors of the building. The receivers were placed on-board of two UAVs, located along the longest and shortest side of the building, at a distance of $10.5 \mathrm{~m}$ and $12.5 \mathrm{~m}$ from the external walls. The UAVs are hovering at an height of 20 $\mathrm{m}$ above ground, i.e., $4 \mathrm{~m}$ over the building rooftop.

\section{A. Coordinate System}

The built-in spatial coordinate system of the ray-tracing simulator is a cartesian one with its origin at ground level and in the centre of the building. For sake of clarity, we adopted a different system with the new origin in the position of each UAV (see Fig. 2) and used spherical coordinates. Moreover, when studying the link between a certain transmitter and a UAV, we rotated the axes in a way that the new $Z$-axis coincides with the LOS path between the transmitter and the UAV. This is especially useful when one needs to evaluate the distribution of the angles of arrivals of the received rays with respect to the LOS direction.

\section{B. Operation Frequency}

It is well known that moving to higher frequencies of the microwave spectrum reduces the capability of the electromagnetic field to pass through walls. As a matter of fact, wave penetration depends on the electromagnetic properties 


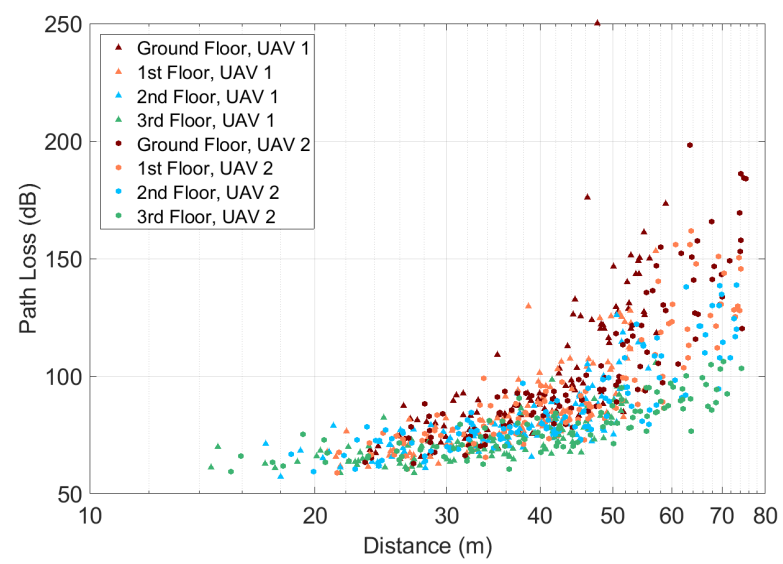

Fig. 3. Path Loss against distance

of the material, i.e., permittivity and conductivity. This has a strong impact on the choice of the most suitable frequency according to the type of environment. For instance, a low frequency $(450 \mathrm{MHz})$ is suitable for countryside coverage, GSM $900(900 \mathrm{MHz})$ for light urban coverage while higher frequencies offer advantages in dense urban coverage in terms of capacity [16]. Today the majority of mobile phones support the use of multiple bands, as well as multi-mode operation. In order to face with the presence of multiple walls that reduce wave penetration, we chose for our simulations a $1800 \mathrm{MHz}$ frequency band (DCS 1800).

\section{Antennas and waveforms}

Antennas and waveform properties are summarized in Table I. We assumed isotropic antennas on both the transmitter and receiver side. A linear horizontal polarization was considered, i.e., the electric field vector of the electromagnetic wave is parallel to the ground. An important point is the polarization matching of the RF antenna on-board the UAV with the incoming signal to get the maximum signal strength. In the simulations we assumed perfect matching, that is, the power is not reflected back from the antenna. Finally, as the simulations are aimed at path-loss evaluation, we considered sine-waves rather than large-band signals.

\section{Numerical Results}

Figure 3 shows the scatterplot of the path loss against the distance between the UT and the UAVs. The measurement points (i.e., the $400 \times 2=800$ possible links) have been divided by floor using four different colors and by UAV using two different markers. It is apparent that UTs corresponding to different floors fill different regions of the plane. Specifically, the path loss increases as the difference between UAV and UT height increases. On the other side, as somewhat expected, the UAV position does not matter (triangles and circles of a color are mixed together). Path loss exhibits considerable variations, ranging from slightly less than $60 \mathrm{~dB}$ on third floor up to more than $150 \mathrm{~dB}$ on ground floor, including an outlier
TABLE II

PATH-LOSS EXPONENT $n$ OF THE MODEL IN (1).

\begin{tabular}{|l||l|}
\hline Floor & $n$ \\
\hline Ground & 19.41 \\
\hline 1st & 13.71 \\
\hline 2nd & 9.73 \\
\hline 3rd & 5.44 \\
\hline
\end{tabular}

as high as $250 \mathrm{~dB}$. Finally, there are huge differences between points located at a similar distance from the UAV.

\section{A. Path Loss Models}

Path Loss (PL) models used in the literature often take the following form [14]:

$$
P L(d)=10 n \log _{10} d+B+C \log _{10} f_{c}+L\left(p_{1}, p_{2}, \ldots, p_{K}\right),
$$

where the coefficient $n$ is often referred to as the path-loss exponent, or power loss coefficient, $B$ is the intercept, $C$ accounts for the frequency dependence and, finally, $L$ is an extra loss dependent on the propagation scenario through the parameters $p_{1}, p_{2}, \ldots, p_{K}$. Though not explicit in (1), $L$ might be distance-dependent as well through the above parameters (e.g., number of walls penetrated in an indoor scenario). $B$ is the path loss value at a distance of $1 \mathrm{~m}$ from the transmitter and at a frequency equal to $1 \mathrm{GHz}$ when $d$ and $f$ are measured in the above units.

It sounds reasonable to study the pattern of path loss against the distance looking at each floor separately, as shown in Fig 4. A linear best fit between $P L$ and $10 \log _{10} d$ has been calculated and drawn in Fig 4. The value of the path-loss exponent is shown in Table II. Note that the slope of the curves is very much different when we move from a floor to another. It increases from about 5.5 (i.e., slightly more than the value $n=4$ of the classical propagation model based on a direct ray and a ground reflected ray) up to about 19 moving from the third floor down to the ground floor. The path loss exponent increases by a factor 4 going from $3^{\text {rd }}$ to $2^{\text {nd }}$ floor and from $2^{\text {nd }}$ to $1^{\text {st }}$, whereas the increase is about 5.5 from $1^{\text {st }}$ floor to ground floor. Finally, note that a simple linear fit is not the most accurate to model the distance dependence in the simulated propagation scenario, especially when we consider the lower floors. Data highlight a change in slope at a breakpoint distance between 40 and $50 \mathrm{~m}$ from the UAV.

The complexity of the simulated environment can be modelled by using an extra loss term $L$ in (1), which is dependent on the number of obstacles encountered along the LOS path between the transmitter and the receiver. Figure 5 shows the PL dependence on the number of walls (both internal and external) and floors. For sake of clarity, the measurement points have been slightly shifted on the $x$-axis to highlight the differences between points that belong to different floors (i.e., different colors). Even though there is a direct dependence of the PL on the number of walls and floors, the scatter is quite large. Moreover, the number of penetrated floors rather than the number of walls has the major impact on PL increment. 


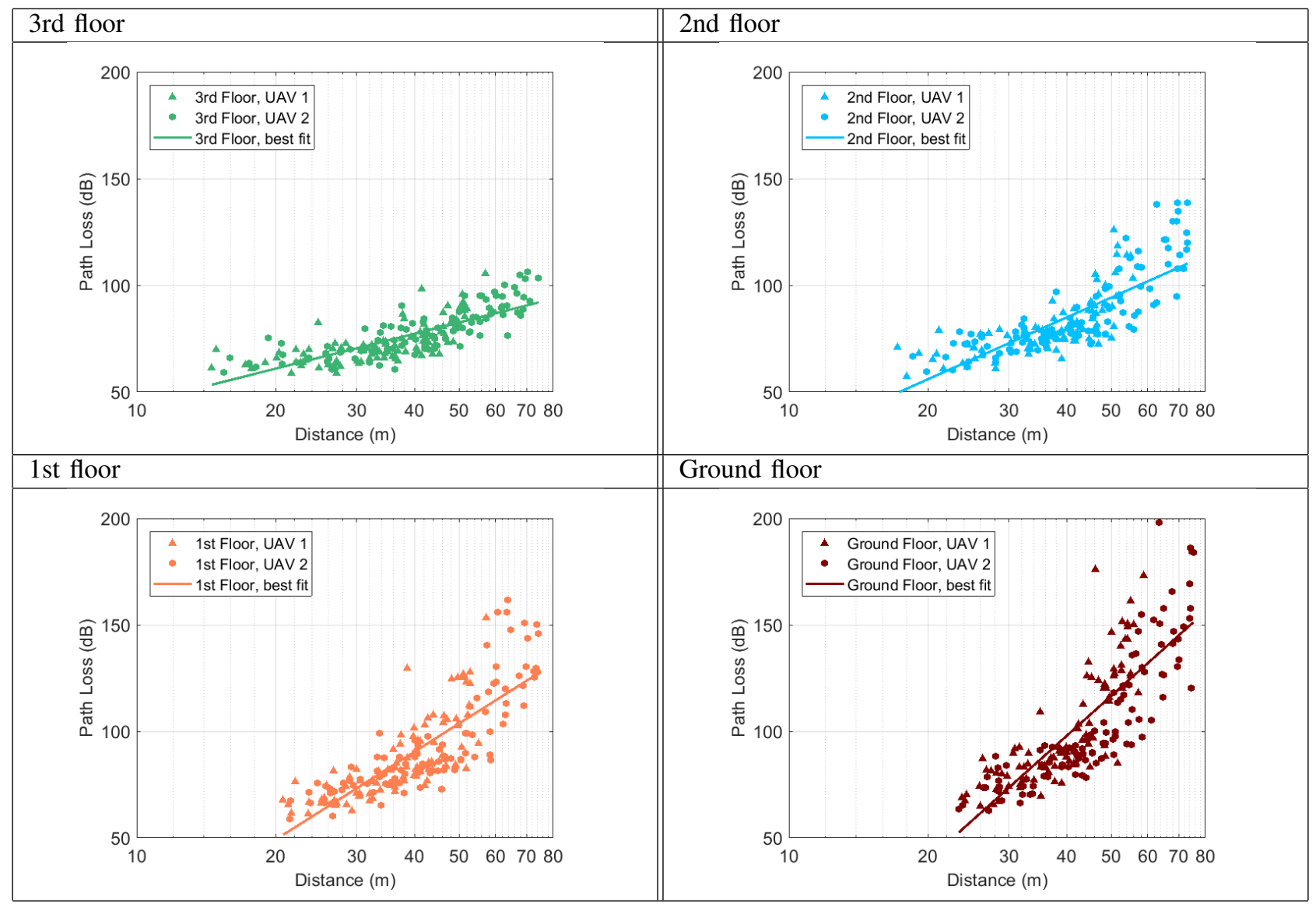

Fig. 4. Path loss against UT-UAV distance for every floor and linear best fit between path loss and logarithm of distance.

This effect is due to the different thickness and material of floors and walls (see Table I).

\section{B. Ray tracing simulation vs models}

Let us consider a few PL models available in the open literature. Usually, the propagation environments are classified into a limited number of possible scenarios. When (1) is adopted, specialized coefficients are tabulated for every scenario [14]. Alternatively, ad-hoc formulas are provided. The above coefficients are either best-fit products over a set of measurements or they are gathered from simple propagation models (e.g., direct+reflected ray model) assuming transmission and reflection coefficients of typical building materials.

On paper, the simulated environment is indoor-to-outdoor. However, the outdoor part of propagation is marginal as the UAV is located close to the external wall of the building with no obstacles in between. Hence, apart from the free-space loss [17], the outdoor path does not need any special modeling. Indeed, an analysis of the angle of arrivals of the rays at each UAV shows that most of contributions reach the UAV after having penetrated the external walls (or windows). There are only a few cases where contributions from ground-reflected rays are received. Finally, the external wall is made of almost non-absorbing bricks (conductivity equal to $0.001 \mathrm{~S} / \mathrm{m}$ ), hence as for propagation, it is not much different from an internal wall. All the above characteristics suggest to compare the simulation results with existing models for indoor propagation.

Specifically, here we consider two indoor models, namely the one for scenario A1 (indoor office) taken from the WinnerII package [14] and the ITU-R path-loss model for indoor scenarios described in Recommendation ITU-R P.1238 [18]. Finally, as a reference lower bound, the simple free-space model (also referred to as Friis equation) is considered as well. All the above models can be formulated through (1), with the coefficient values as illustrated in Table III. WinnerII model takes into account both wall and floor penetration loss, whereas ITU-R P.1238 accounts only for the latter. The two expressions for the floor penetration loss are very similar.

Figure 6 adds to the simulation results the four model curves (i.e., LOS, ITU-R and Winner-II) as black, red and magenta lines, respectively. ITU-R and Winner-II model have been evaluated using the parameters of the actual UT, i.e., the number of penetrated walls $\left(N_{w}\right)$ and floors $\left(N_{f}\right)$. This is the reason of the saw-tooth patterns in the figure. As for the ITU$\mathrm{R}$ and Winner-II models, we assumed that if $N_{f}=0$, the term in the last column of Table III is zero (rather than $15 \mathrm{~dB}$ and $17 \mathrm{~dB}$ respectively). Please note that Winner-II model has a path loss exponent close to -4 in the NLOS case instead of -2 , which is the reason why it starts with such a large PL 

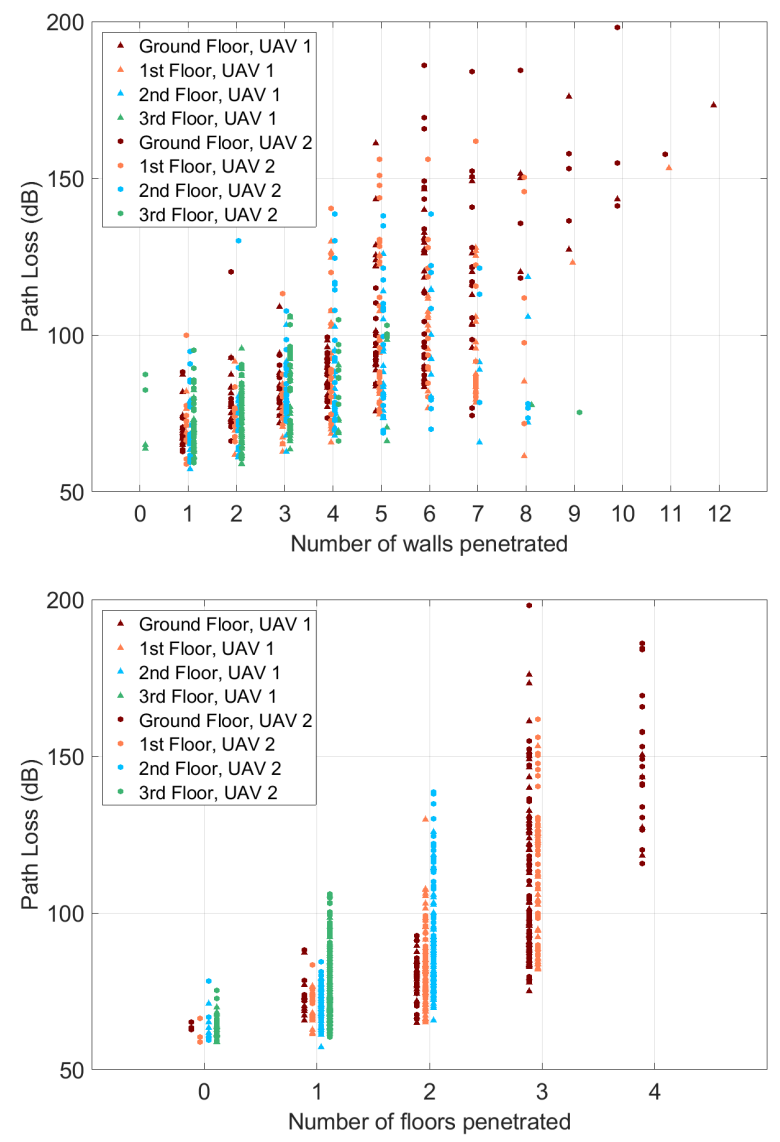

Fig. 5. Path loss against the number of walls penetrated (top panel) and the number of floors penetrated (bottom panel).

TABLE III

COEFFICIENTS OF (1) FOR DIFFERENT PROPAGATION MODELS ASSUMING ISOTROPIC ANTENNAS AND AN OPERATION FREQUENCY OF $1.8 \mathrm{GHZ}$.

\begin{tabular}{|c|c|c|c|c|c|}
\hline \multicolumn{2}{|l|}{ Model } & $n$ & $B$ & $C$ & $L\left(p_{k}\right)$ \\
\hline \multicolumn{2}{|l|}{ LOS } & 2 & 32.4 & 20 & 0 \\
\hline \multicolumn{2}{|c|}{$\begin{array}{l}\text { ITU-R P.1238-10 } \\
\text { (indoor-office) }\end{array}$} & 3 & 32.4 & 20 & $\begin{array}{l}15+4\left(N_{f}-1\right), \text { where } \\
N_{f} \geq 1 \text { is the number of } \\
\text { floors between UT and BS }\end{array}$ \\
\hline \multirow[b]{2}{*}{$\begin{array}{l}\text { Winner-II } \\
\text { A1 } \\
\text { scenario } \\
\text { (indoor } \\
\text { office) }\end{array}$} & LOS & 1.87 & 32.8 & 20 & 0 \\
\hline & NLOS & 3.68 & 29.8 & 20 & $\begin{array}{l}17+4\left(N_{f}-1\right)+5\left(N_{w}-1\right) \text {, } \\
\text { where } N_{w} \geq 1 \text { is the num- } \\
\text { ber of internal walls be- } \\
\text { tween UT and BS and } N_{f} \\
\text { as above }\end{array}$ \\
\hline
\end{tabular}

value at the points of the third floor close to the wall, where the UAV is placed ( $N_{w}=1$ and $\left.N_{f}=0\right)$. The black-line highlights that the PL in this case is of the same order as the one in free-space as the transmission loss through the external wall is not significant. By passing, we note that the PL can be less than the one in free-space because, for non-grazing incidence, the phase of the reflection coefficient is close to 0 , hence reflected rays add in phase to the direct ray.

If we neglect (or adjust) the extra loss of the Winner-II model in "near LOS" conditions, the slope of the corresponding curves follows much better the one of the simulation points especially in the cases of $1^{\text {st }}$ floor and ground floor. On the other side, the ITU-R model looks too rough and does not describe the complexity of the environment. In fact, it does not account for the implicit distance-dependence introduced when the number of walls between UT and UAV increases.

\section{CONClusion}

Through ray-tracing simulations, we quantified the path loss experienced by a signal transmitted from indoor user terminals located inside a building to a UAV in proximity of it. An overall number of 400 transmitters was randomly deployed within a $60 \times 40 \mathrm{~m}$ four-floor building with two UAVs acting as receivers midway through its major and minor side at about $10 \mathrm{~m}$ from the external wall and at an height of $20 \mathrm{~m}$. The calculated path loss shows a complex dependence on the transmitter-UAV distance, as well as a severe increase when the number of floors between the terminals and the UAV increases.

Path loss models available in the literature for indoor propagation, which is a scenario similar to the one we simulated, are not able to fully capture the characteristics of this particular environment. For instance, the ITU-R model takes into account only through floor propagation losses while neglecting internal walls, which results in a too smooth dependence of the path loss on the distance. On the other side, the Winner-II model, when re-normalized to match path loss values close to the UAV, fits better the slope of the curve.

Future work will be devoted to the definition of a path loss model for this propagation scenario, taking into account a number of UAV positions outside the building and directional antennas. Besides the definition of the position of the UAV, more realistic distributions of the users in emergency situations will be investigated. This could lead to the development of algorithms for the localization of trapped users. Finally, we point out that the building materials and their electrical properties were taken from the internal database of the raytracing simulator. Further developments shall also include a test of path loss sensitivity to different materials (for instance using the data provided by ITU-R P.2040-1).

\section{REFERENCES}

[1] Y. Lin, T. Wang, and S. Wang, "UAV-assisted emergency communications: an extended multi-armed bandit perspective," IEEE Communications Letters, vol. 23, pp. 938-941, 2019.

[2] D. Wang, B. Bai, G. Zhang, and Z. Han, "Optimal placement of lowaltitude aerial base station for securing communications," IEEE Wireless Communications Letters, vol. 8, 869-872, 2019.

[3] Z. Na, B. Mao, J. Shi, J. Wang, Z. Gao, and M. Xiong, "Joint trajectory and power optimization for UAV-relay-assisted Internet of Things in emergency," Physical Communication, Article 101100, Aug. 2020.

[4] J. Cui, B. Hu, and S. Chen, "Resource allocation and location decision of a UAV-relay for reliable emergency indoor communication," Computer Communications, 2020.

[5] B. Li, Z. Fei, and Y. Zhang, "UAV communications for 5G and beyond: Recent advances and future trends," IEEE Internet of Things Journal, pp. 2241-2263, 2018.

[6] C. Yan, L. Fu, J. Zhang, and J. Wang, "A comprehensive survey on UAV communication channel modeling," IEEE Access, vol. 7, 107769107792, 2019. 


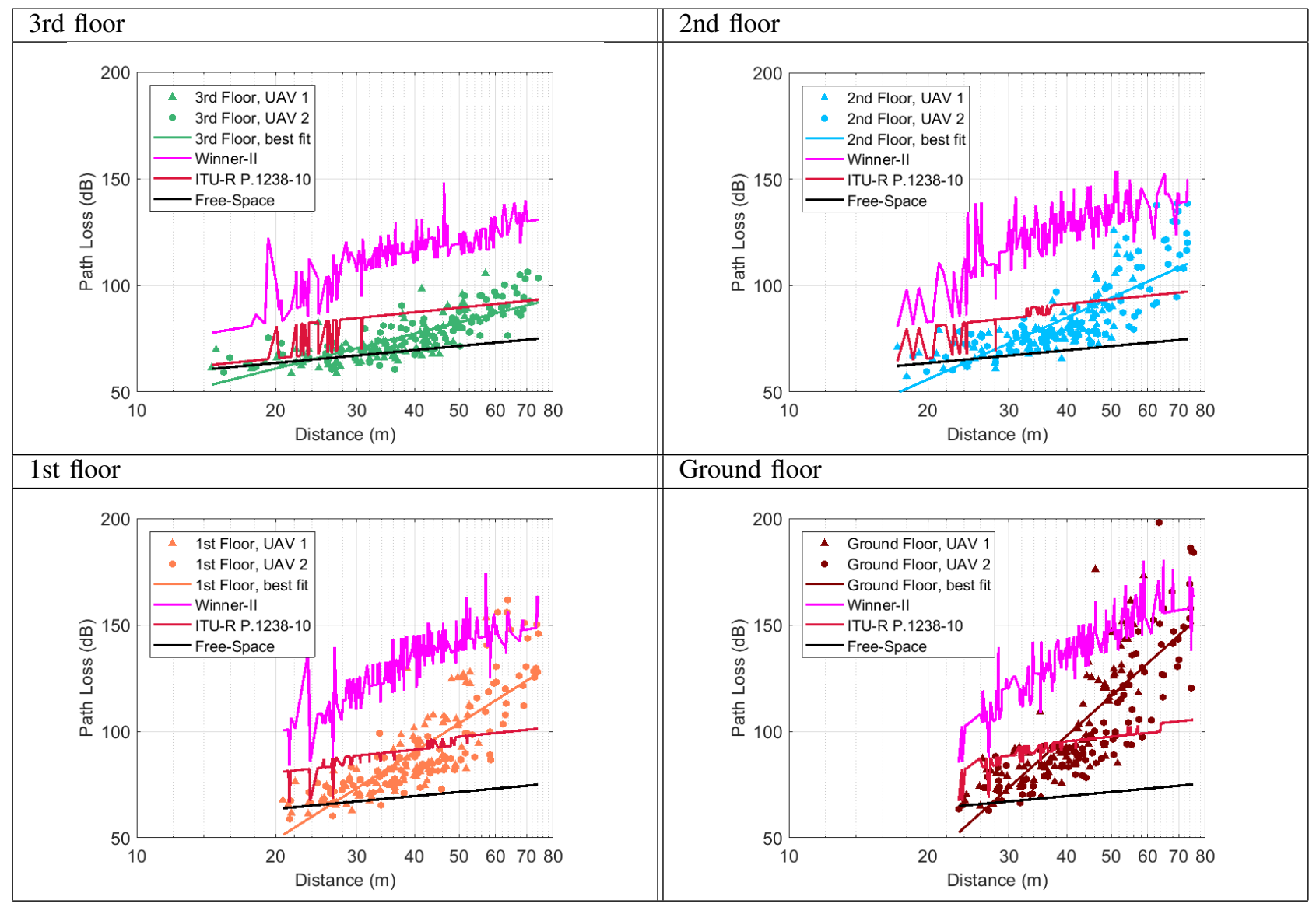

Fig. 6. Path loss against UT-UAV distance for every floor: comparison between simulations and three different models.

[7] S. Hamid, A. Al-Dweik, M. Mirahmadi, K. Mubarak, and A. Shami, "Inside-out propagation: Developing a unified model for the interference in 5G networks," IEEE Vehicular Technology Magazine, vol. 10, pp. 47-54, June 2015.

[8] P. Rademacher. Ray tracing: Graphics for the masses. [Online]. Available: https://www.cs.unc.edu/ rademach/xroads-RT/RTarticle.html

[9] K. Ukhurebor and C. Abiodun, "Assessment of building penetration loss of cellular network signals at $900 \mathrm{mhz}$ frequency bands in Otuoke, Bayelsa State, Nigeria," 2018.

[10] Q. Feng, J. Mcgeehan, E. Tameh, and A. Nix, "Path loss models for airto-ground radio channels in urban environments," 2006, pp. 2901-2905.

[11] Q. Feng, E. Tameh, A. Nix, and J. Mcgeehan, "Modelling the likelihood of line-of-sight for air-to-ground radio propagation in urban environments," Proc. of Globecom, 2007, pp. 1-5.

[12] A. Al-Hourani, K. Sithamparanathan, and A. Jamalipour, "Modeling airto-ground path loss for low altitude platforms in urban environments," 2014 IEEE Global Communications Conference, pp. 2898-2904, 2014.

[13] Remcom Inc, "Wireless InSite Reference Manual," version 3.3.3, May 2019.

[14] J. Meinila, P. Kyosti, T. Jamsa, and L. Hentila, "WINNER II channel models" in Radio Technologies and Concepts for IMT-Advanced, pp. 39-92, 2009.

[15] K. P. Thakur and W. S. Holmes, "Reflection of plane wave from multilayered dielectrics," Proc. of APMC, vol. 2, 2001, pp. 910-913.

[16] S. J. P. T. Joseph and S. Mohapatra, "Managment information system in the knowledge Economy," PHI Learning Pvt. Ltd., pp. 384-386, 2014.

[17] J. D. Parsons, The Mobile Radio Propagation Channel, Second Edition, J. Wiley \& Sons, 2001, Print ISBN:9780471988571 —Online ISBN:9780470841525 —DOI:10.1002/0470841524

[18] Recommendation ITU-R P.1238, "Propagation data and prediction methods for the planning of indoor radiocommunication systems and radio local area networks in the frequency range $300 \mathrm{MHz}$ to $450 \mathrm{GHz}$ ". 